\title{
Mercury's Low Reflectance Material - Evidence for Graphite Flotation in a Magma Ocean?
}

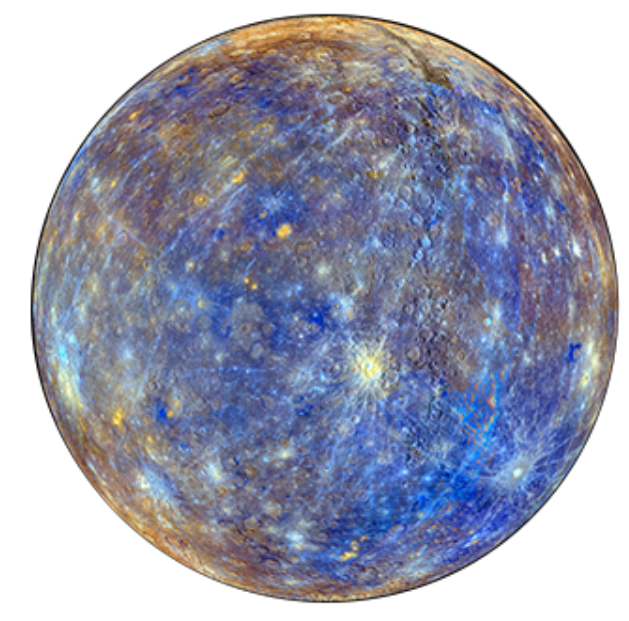

\section{Lead Author:}

Rachel L. Klima

Johns Hopkins Applied Physics Laboratory, Laurel, MD

Rachel.Klima@jhuapl.edu

443-778-0627

\section{Co-Authors:}

Carolyn Ernst, Johns Hopkins Applied Physics Laboratory

Nancy Chabot, Johns Hopkins Applied Physics Laboratory

Kathleen Vander Kaaden, Jacobs/NASA Johnson Space Center

Sebastien Besse, European Space Agency

Marc Fries, NASA Johnson Space Center

\section{Co-Signers:}

David Blewett, Johns Hopkins Applied Physics Laboratory; Paul Byrne, North Carolina State University; Joshua Cahill, Johns Hopkins Applied Physics Laboratory; Christian Carli, Institute for Space Astrophysics and Planetology, INAF; Brett Denevi, Johns Hopkins Applied Physics Laboratory; Ariel Deutsch, Brown University; Cesare Grava, Southwest Research Institute; Benjamin Greenhagen, Johns Hopkins Applied Physics Laboratory; Jennifer Grier, Planetary Science Institute; Steven Hauck, II, Case Western Reserve University; James Head, Brown University; Jörn Helbert, DLR Institute of Planetary Research; Hauke Hussmann, DLR Institute of Planetary Research; Suzanne Imber, University of Leicester; Catherine Johnson, Planetary Science Institute \& University of British Columbia; Lauren Jozwiak, Johns Hopkins Applied Physics Laboratory; Christian Klimczak, University of Georgia; Francis McCubbin, NASA Johnson Space Center; Karen Stockstill-Cahill, Johns Hopkins Applied Physics Laboratory; Faith Vilas, Planetary Science Institute; Jennifer Whitten, Tulane University 


\subsection{Introduction}

Mercury was revealed by the MESSENGER mission to be an enigmatic and exciting planet. Its giant iron core and extraordinarily low-iron surface suggest that the raw materials that accreted and differentiated into Mercury were extremely oxygen-deprived, with an environment 3-5 orders of magnitude more reducing than that in which the Moon formed. Contrary to expectations based on early formation models for Mercury, instruments on board MESSENGER revealed a surface relatively rich in volatile elements, including sulfur and potassium. Landforms, including pyroclastic deposits and vents and enigmatic 'hollows'-bright features thought to have formed by collapse due to loss of volatiles-are scattered across the planet. The

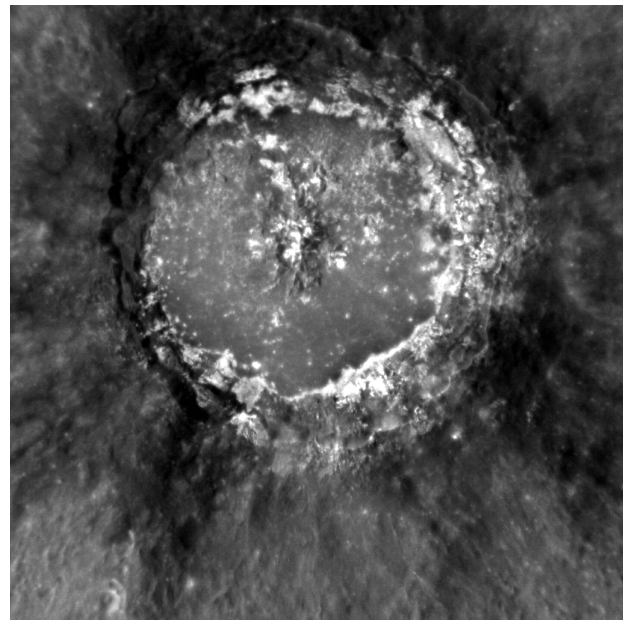

Fig. 1. Bright hollows in Basho crater are associated with LRM. average albedo of the crust is extremely low relative to other low-iron surfaces, such as the lunar highlands. Low reflectance material (LRM), which exhibits an albedo even lower than that of the average crust of Mercury, is exhumed by craters and found in patches throughout the ancient intercrater plains. In many locations, there is evidence of hollows developing within these LRM deposits (Fig. 1; Solomon et al., 2018).

Despite the wealth of information gathered by the MESSENGER mission, the mineralogy of Mercury's surface largely remains a mystery. Diagnostic crystal field absorption bands caused by ferrous iron in silicates are weak or absent from the visible and near-infrared (NIR) spectra of Mercury, suggesting that the ferrous iron content of silicates at the surface is extremely low (e.g., Izenberg et al., 2014, Murchie et al., 2015). Elemental measurements confirm an extremely low abundance of iron in Mercury's crust (Nittler et al., 2011; Evans et al., 2012; Weider et al., 2014), and, based on visible and NIR measurements, the small amount of iron that is detected may be contained in sulfides or even reduced as iron metal. While BepiColombo will surely provide new and critical information about surface mineralogy, particularly in the infrared wavelength range, the harsh space weathering environment will likely continue to complicate spectral analysis.

Visible to NIR reflectance spectra of LRM exhibit lower albedo and a flatter slope than the average reflectance for Mercury, which is dark but strongly red sloped. In some cases, LRM spectra also exhibit a curvature suggestive of a weak, broad absorption band near $600 \mathrm{~nm}$ (Robinson et al., 2008; Murchie et al., 2015). From Mariner 10 and early MESSENGER flyby observations, it was suggested that ilmenite, ulvöspinel, carbon, or Fe metal could cause the characteristic dark, flat spectrum of LRM and the global darkening of Mercury (Robinson et al., 2008). Given the very dark nature of LRM, it was initially proposed that it could be the result of extensive space weathering (Riner and Lucey, 2012). Once MESSENGER entered orbit, low Fe and Ti abundances measured by the X-Ray and Gamma-Ray Spectrometers (XRS and GRS) ruled out ilmenite and ulvöspinel as important surface constituents (Nittler et al., 2011; Evans et al., 2012) and implied that LRM was darkened by a different phase, such as carbon or small amounts of micro- or nanophase opaque minerals dispersed in a silicate matrix. 
Targeted, low-altitude thermal neutron measurements for three LRM-rich regions confirmed an enhancement of 1-3 wt\% carbon over the global abundance, supporting the hypothesis that the darkening agent in LRM is carbon (Peplowski et al., 2016). Though seemingly unlikely on a terrestrial planet's surface-particularly one so close to the Sun-two explanations for carbon on Mercury's surface had been proposed. The first suggests that carbon could be exogenic, delivered gradually by comets over Mercury's history (Bruck Syal et al., 2015). The second suggests an endogenic origin, that any carbon that did not partition into the core of the planet would crystallize as graphite, and would have floated to the surface creating a primordial graphite flotation crust (Vander Kaaden and McCubbin, 2015). Across the surface of Mercury, LRM shows clear evidence of having been excavated from depth (Ernst et al., 2015; Murchie et al., 2015; Klima et al., 2018). In cases where it is not clearly associated with specific craters, it occurs in patchy spots within broad regions of heavily cratered, ancient terrain where the ejecta from numerous small craters overlap (Denevi et al., 2016; Leeburn et al., 2017). This evidence, derived from globalscale mapping efforts, appears to support the hypothesis that this carbon may be sourced from the remnants of a magma ocean flotation crust. However, many questions remain.

\subsection{The Mineralogy of Mercury}

Resolved measurements of the surface using XRS data provide the best direct compositional information about Mercury's surface (Weider et al., 2015). As illustrated in Fig. 2, Al and $\mathrm{Mg}$ are nearly, but not completely, anticorrelated with high Al and lower $\mathrm{Mg}$ in the smooth plains of Caloris (which are also brighter and somewhat redder relative to average Mercury), and low $\mathrm{Al}$ and high $\mathrm{Mg}$ in the 'Mg-rich region' near $270^{\circ} \mathrm{E}$. Weider et al. (2015) also presented XRS flare data for $\mathrm{Ca}$ and $\mathrm{S}$, showing a relationship between both of those elements and the Mg-rich region; however, coverage is limited due to the distribution of flare data. Fe data are even more limited, but the few available flare swaths suggest that it, too, may be correlated with the Mgrich region.

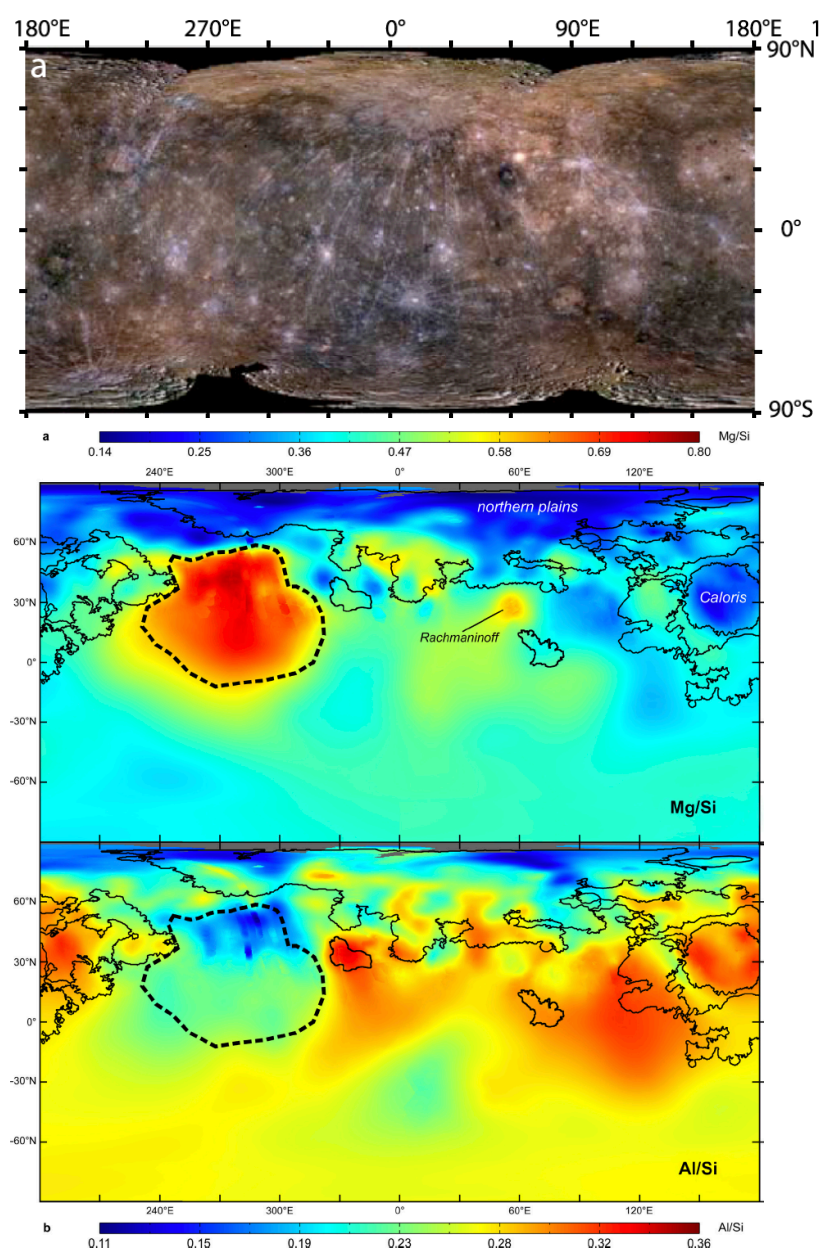

Fig. 2. (top) Simple cylindrical projection of Mercury MDIS data with the $1000 \mathrm{~nm}$ band shown in red, $750 \mathrm{~nm}$ band shown in green, and 430 band shown in blue (from Klima et al., 2018). (bottom) Simple cylindrical projection of $\mathrm{Mg} / \mathrm{Si}$ and $\mathrm{Al} / \mathrm{Si}$ abundance as measured by the XRS (from Weider et al., 2015). 
Several general assumptions about the global mineralogy can be made based on the elemental measurements, but much uncertainty remains. The anticorrelation between $\mathrm{Ca}$ and $\mathrm{Al}$ suggests that plagioclase is largely albitic. The very mafic Mg-rich region is likely associated with sulfides containing $\mathrm{Mg}$, Fe, and $\mathrm{Ca}$ similar to oldhamite or niningerite found in highly reduced meteorites. However, it is important to remember that the spatial resolution of these data is extremely coarse, and that, except for the case of the high reflectance plains (HRP), there is little to no relationship between the geochemical terranes and the geological or color properties. The smooth northern volcanic plains are uniformly lower in $\mathrm{Mg}$ than some of the rest of the planet, but they are not uniformly enriched in Al (Fig. 2). Variations observed in the southern hemisphere rely on only a few flares that were observed while MESSENGER was far from the planet's surface, and thus represent very large spatial footprints.

Vander Kaaden et al. (2017) used the available elemental data to compute the cation norms for nine geochemical regions, and their results showed that the HRP contain $\sim 58 \%$ plagioclase (likely albitic) and a mixture of low and high-Ca pyroxene, with almost $8 \%$ quartz in Caloris. Conversely, the high-Mg region is interpreted to contain significant ( $27 \%)$ olivine and just under $41 \%$ plagioclase, with over $5 \%$ sulfides. The bulk of the rest of the planet-the intermediate terrainis modeled to contain just over $50 \%$ plagioclase, $\sim 4 \%$ sulfides and most of the rest low-Ca pyroxene. While these values provide an important basis for understanding the evolution of volcanism on Mercury, they are just a first step, and convolve many assumptions. One among them is that in order to perform these calculations, one assumes the valence state of each of the element, and that sufficient oxygen is present to balance the equations. However, direct measurements of $\mathrm{O}$ on Mercury's surface by the GRS report a lower O/Si weight ratio than necessary to balance these equations (Evans et al., 2012; McCubbin et al., 2017). McCubbin et al. (2017) suggest that this may be due to smelting of silicates during eruption through a graphite layer. Taken together, the coarse spatial footprints, potential smelting at the surface, and lack of global coverage for critical elements such as $\mathrm{Fe}$ and $\mathrm{Ca}$ result in large uncertainty in the mineralogy of volcanic flows and key geological features on Mercury that require extensive investigations in the next decade.

\subsection{Low Reflectance Material: Carbon- Rich, Ancient Terrain}

LRM is distributed across Mercury, typically having been excavated from depth by craters and basins (Figs. 3, 4). In contrast to the brighter HRP and smooth plains deposits, which exhibit morphological evidence of volcanism (e.g., Head et al, 2011; Denevi et al, 2013;

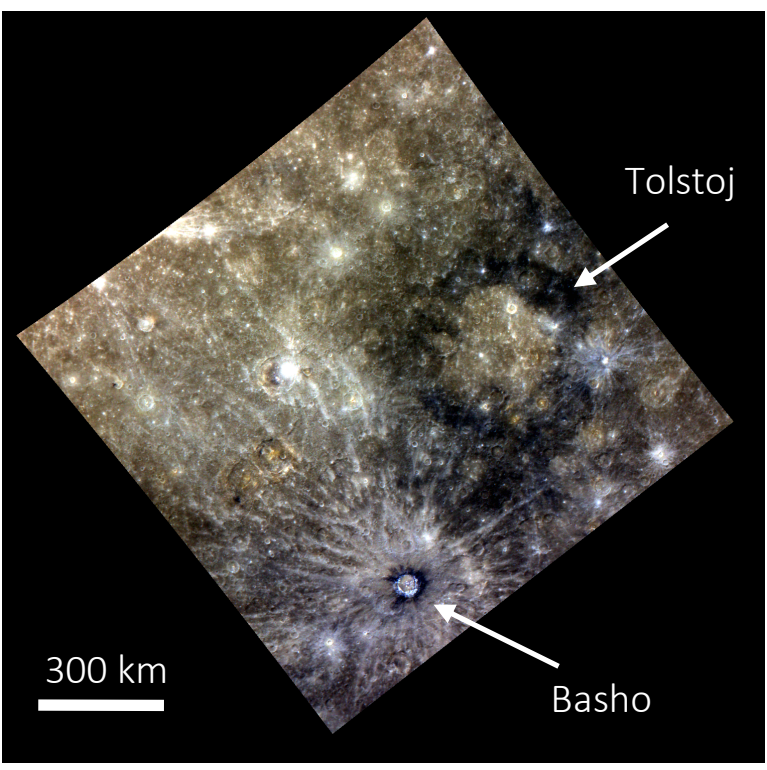

Fig. 3. LRM exposed at Tolstoj (centered at $16.3^{\circ} \mathrm{S}, 163.5^{\circ} \mathrm{W}$ ) and Basho Craters. Material exposed around the older, larger Tolstoj grades into the surrounding intercrater plains, while the younger Basho only clearly excavates LRM within the proximal ejecta. 


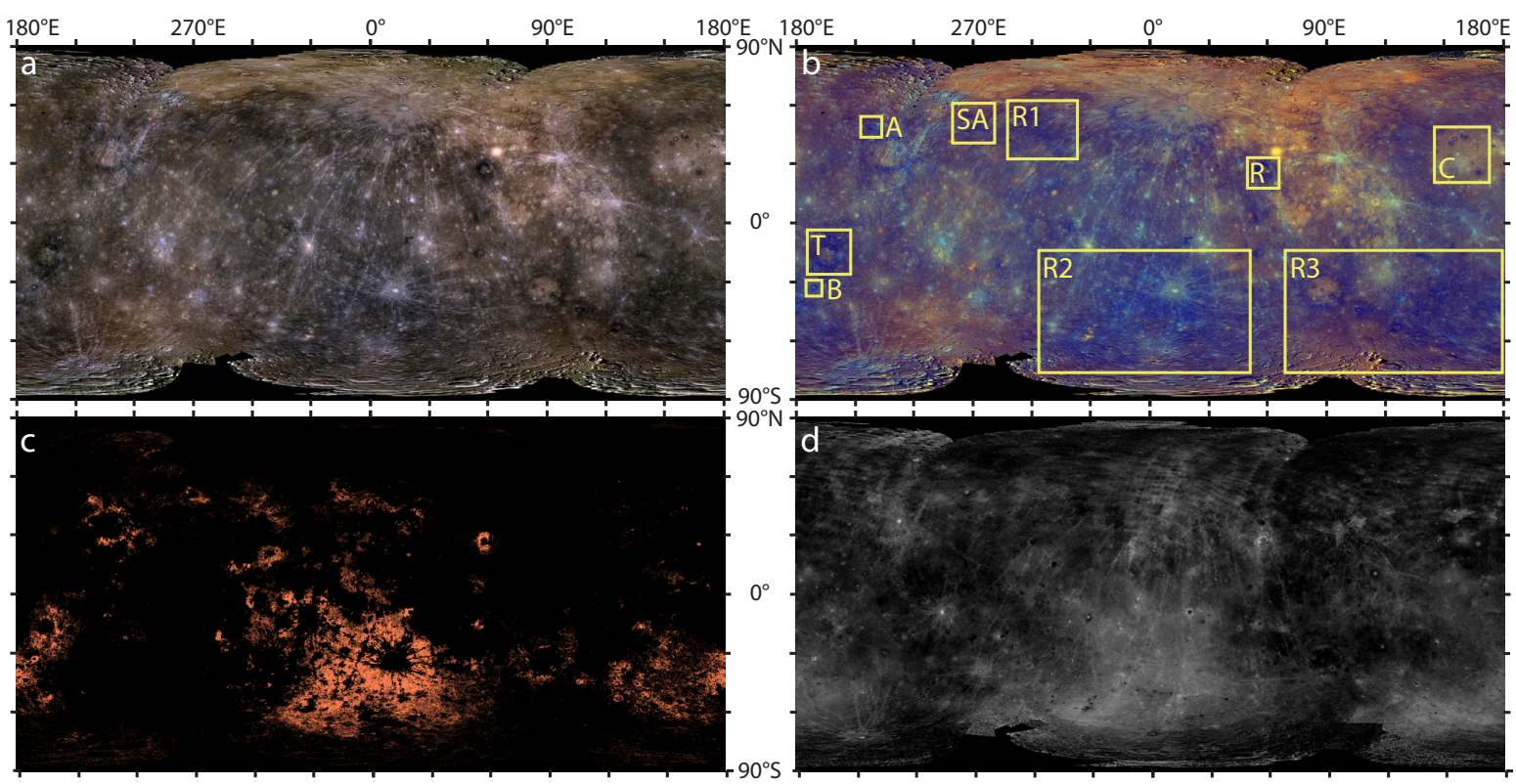

Fig. 4. (a) False color global map of Mercury with $R=1000 \mathrm{~nm}, \mathrm{G}=750 \mathrm{~nm}$ and $B=430 \mathrm{~nm}$. LRM shows up as dark bluish-black material, and grades into the slightly brighter LBP.

(b) Enhanced color composite of Mercury with R=PC1, G=PC2, B=430/1000 nm slope. LRMenriched craters T-Tolstoj, B-Basho, A-Akutagawa, SA-Sholem-Aleichem, R-Rachmaninoff and C-Craters within Caloris are outlined in yellow, as are three regional enhancements (R1, R2, R3). (c) LRM map using albedo, PC2 and slope constraints as detailed in the text. (d) 600-nm broad band depth map (figure from Klima et al., 2018).

Whitten et al., 2014), LRM is not associated with flow features or other evidence of a volcanic origin. Older LRM boundaries are generally diffuse, and grade into low reflectance blue plains (LBP). Because of the common lack of sharp geologic boundaries, LRM has been defined primarily based on albedo and spectral shape, isolated through principal components (PC) analyses of MDIS color images (Klima et al., 2018). The low iron content of Mercury's surface results in a lack of the spectral absorption bands typically used to map mafic minerals on planets and asteroids. Therefore, mathematical transformations such as PC analysis are used to tease apart subtle spectral differences. The second principal component (PC2), which captures a combination of spectral slope and curvature, isolates LRM and hollows as one endmember, with red material and HRP as the other end member. LBP and intermediate plains (IP) are transitional from LRM to HRP (Denevi et al., 2016). Concentrated LRM exposures have been defined as regions with a photometrically corrected reflectance of $<5 \%$ (at $560 \mathrm{~nm}$ wavelength) and a PC2 value in the lower $25 \%$ of the range of PC2 values for the whole planet (Peplowski et al., 2016).

\subsection{A Mercurian Magma Ocean}

It has been suggested that Mercury differentiated through a magma ocean stage in which substantial heating and melting of the planet took place (Brown and Elkins-Tanton, 2009; Riner et al., 2009). On the Moon, we see evidence of a global lunar magma ocean (LMO) through the presence of a primary anorthositic crust. According to existing LMO crystallization models, plagioclase began to crystallize after about $75 \%$ crystallization (Snyder et al., 1992) and was 
buoyant with respect to the FeO-rich residual melt, leading to plagioclase flotation and the formation of a primary anorthositic flotation crust (Jolliff et al., 2000). Vander Kaaden and McCubbin (2015) showed that the low FeO content and limited density range of mercurian melts prohibits nearly all rock-forming minerals from forming a primary flotation crust on Mercury with the exception of graphite (Fig. 5). Furthermore, Mercury's shallow mantle results in a limited pressure and temperature profile that does not span into the diamond stability field. Graphite is therefore the stable phase of elemental carbon throughout the silicate portion of Mercury, which is a primary prerequisite to the existence of a graphite flotation crust.

a.

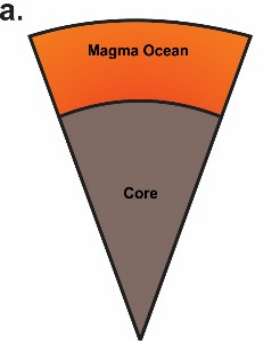

b.

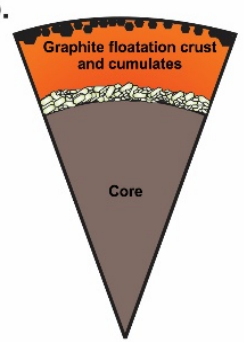

C. Volcanic secondary crust

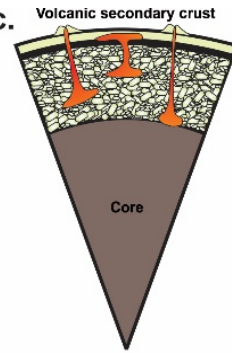

d.

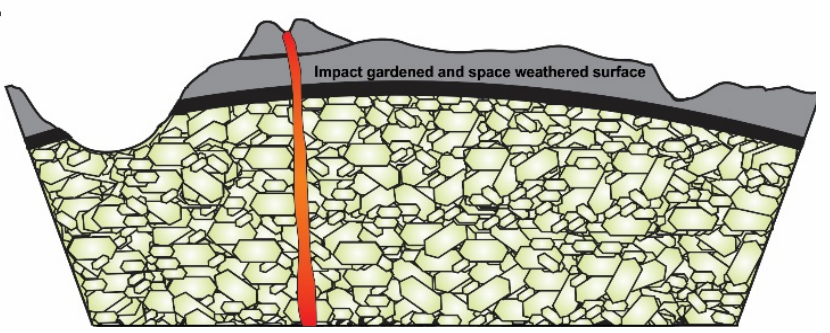

Fig. 5. Possible stratigraphy of Mercury after graphite flotation in a magma ocean and subsequent volcanism and cratering (from Vander Kaaden et al. 2019).

Although geochemically plausible, the physical existence of a primary graphite flotation crust on Mercury, as well as the implications for planet wide distribution and associated magma ocean crystallization, have yet to be explored. Assuming a graphite flotation crust was present, the thickness and extent of that crust would be dictated by the amount of carbon allocated to the silicate portion of the planet and the efficiency of graphite flotation. These parameters are critical to place further constraints on the thermochemical evolution of Mercury and warrant additional exploration over the next decade.

\subsection{Mercury: A Compelling Target for Understanding Solar System Evolution}

Most of our understanding of the early thermal and chemical evolution of terrestrial planets is based on the LMO model (e.g., Wood et al., 1970; Warren, 1990), derived from studies of lunar samples and supported by lunar remote sensing data. Magma ocean models have been applied to bodies including Earth and Mars (e.g., Elkins-Tanton et al., 2003; Elkins-Tanton, 2012), and also large asteroids such as Vesta (e.g., Righter and Drake, 1997). However, only the Moon bears obvious evidence of a planet-wide flotation crust, suggesting that perhaps smaller bodies such as Vesta cooled too quickly for any major minerals to form a primary flotation crust, and that larger bodies such as Earth and Mars may have experienced a significantly more complicated thermal evolution, with multiple magma ocean stages and extensive density-driven convection (e.g., Elkins-Tanton, 2012). Studying Mercury offers a unique opportunity to explore terrestrial planet evolution of another body, somewhat larger ( $700 \mathrm{~km}$ larger in radius) than the Moon, that may be in the "sweet spot" for magma ocean formation and primary crust preservation.

On a broader scale, evidence from MESSENGER suggests Mercury may hold clues to the distribution of elements in the early Solar System. Its high volatile content contradicts earlier models for origin of its large metal to silicate ratio, including that a large impact stripped off much of the rocky shell, or that the crust was lost by evaporation. Understanding the roles of $C$ and 
other volatiles on Mercury is key not only to determining how Mercury formed and evolved, but also to constraining the distribution of materials in our protoplanetary nebula, e.g. the Ebel and Alexander (2011) model of Mercury's accretion in a C-rich inner nebula. Providing further information about whether Mercury had a graphite flotation crust will have implications on models of solar system formation in general, because short-period, C-rich planetesimals are predicted for moderately C-enriched stars (Moriarty et al., 2014), and two of the smallest extrasolar rocky planets for which the mass and size have been determined are also dense and close to their stars (Wurm et al., 2013).

\subsection{Recommendations}

Overall, we recommend that in the next decade, studies of Mercury remain a high priority. Fundamental data analysis, modeling, and analog laboratory research should be supplemented with the opportunity to advance the spacecraft exploration of Mercury. Importantly, we recommend that the decadal survey consider the critical role of team dynamics, equity, diversity, inclusion, and accessibility in planetary science. We strongly encourage the decadal survey to consider the state of the profession and these issues - not as separable matters, but as critical steps on the pathway to facilitating missions and additional laboratory/modeling/analog studies in support of Mercury, our solar system, and beyond. Studies of scientific teams have repeatedly demonstrated the importance of an integrated approach, where team members with diverse expertise develop synergies between their specialties and resources that result in an end product that adds up to more than the sum of its parts (Balakrishan et al. 2011). Sociological studies have demonstrated that groups that foster strong connections across sub-units are more innovative (Burt 2004; Powell and Koput, 1996; de Vaan et al., 2015) with higher impact outcomes that endure (de Vaan et al. 2015, Curral et al. 2001).

Mercury's LRM offers a scientifically compelling and unique opportunity to investigate the earliest stages of planetary evolution and crust formation. We recommend that in situ mineralogical measurements of Mercury's LRM should be a scientific priority in the next decade, to address high priority questions, including:

- Did Mercury have a magma ocean, and, if so, how did its crystallization affect the subsequent evolution of the planet?

- What is the composition of the LRM on Mercury?

- What processes led to the presence of graphite and sulfides contained in the crust?

- What is the extent, preservation, and inferred thickness of a carbon layer on Mercury?

- How much carbon would have had to be available in the silicate portion of Mercury to form the ostensible graphite flotation crust?

- What is the detailed mineral composition of Mercury's surface?

- Did smelting occur on the Mercury's surface?

- Why are the hollows found in association with the LRM, and what other minerals are associated with them?

- What is the composition of the hollows, and how did they form?

- What do volatile abundances of Mercury's crust tell us about volatile distribution of the inner solar system? 


\subsection{References}

Balakrishan et al. (2011) (https://dl.acm.org/doi/10.1145/1958824.1958905).

Brown, S. M., and L. T. Elkins-Tanton (2009), Earth and Planet. Sci. Lett., 286(3-4), 446-455.

Bruck Syal, M. et al. (2015) Nat. Geosci. 8, 352-356.

Burt (2004) (https://www.journals.uchicago.edu/doi/10.1086/421787).

Curral et al. (2001) (https://www.tandfonline.com/doi/abs/10.1080/13594320143000627).

Denevi, B. et al. (2016) LPSC, Abstract No. 1624.

Denevi, B.W. et al. (2013) J. Geophys. Res. 118, 891-907. http://dx.doi.org/10.1002/igre.20075.

de Vaan et al. (2015) (https://www.jstor.org/stable/10.1086/681213?seq=1)

Ebel, D. S. and Alexander, C.M.O'D. (2011). Planet. Space Sci. 59, 1888-1894.

Elkins-Tanton, L. T. (2012) Annu. Rev. Earth Planet. Sci. 40, 113-39.

Elkins-Tanton, L. T. et al. (2003) Meteor. \&Planet. Sci. 38, 1753-1771.

Ernst, C.M., et al. (2015) Icarus, 250, 413-429.

Evans, L.G., et al. (2012) J. Geophys. Res. 117, E00L07, doi:10.1029/2012JE004178.

Head, J.W. et al. (2011) Science 333, 1853-1856. http://dx.doi.org/10.1126/ science.1211997.

Izenberg, N.R., et al. (2014) Icarus, 228, 364-374.

Jolliff, B. L., et al. (2000) J. Geophys. Res., 105, 4197-4216.

Klima, R.L., et al. (2018), Geophys. Res. Lett., 45, 2945-2953.

Leeburn, J. M., et al. (2017) LPSC, Abstract No. 1964.

McCubbin, F.M., et al. (2017) J. Geophys. Res., 122, 2053-2076.

Moriarty, J., et al. (2014) Astrophys. J., 787, article id. 81.

Murchie, S.L, et al. (2015) Icarus, 254, $287-305$.

Nittler, L.R., et al. (2011) Science, 333, 1847-1850.

Peplowski, P. N., et al. (2016) Nature Geoscience, 9, 273-278.

Powell and Koput, (1996) (https://www.jstor.org/stable/2393988?origin=crossref\&seq=1)

Righter, K. and Drake, M. J. (1997) Meteor. \&Planet. Sci., 32, 929-944.

Riner, M. A., et al. (2009), Geophys. Res. Lett, 36, L02201, doi:10.1029/2008GL036128.

Riner, M. A. and Lucey, P. (2012) Geophys. Res. Lett 39, L12201, doi:10.1029/2012GL052065.

Robinson, M.S., et al. (2008) Science, 321, 66-69.

Snyder, G. A., et al. (1992), Geochim. Cosmochim. Acta, 56, 3809-3823.

Solomon, S. C., Nittler, L. R., \& Anderson, B. J. (Eds.). (2018). Mercury: The view after MESSENGER. Cambridge University Press.

Vander Kaaden, K.E., and McCubbin, F.M. (2015). J. Geophys. Res. Planets, 120, 195-209.

Vander Kaaden, K. E., et al. (2017) Icarus 285, 155-68. doi:10.1016/j.icarus.2016.11.041.

Vander Kaaden, K.E., et al. (2019) Space Sci Rev 215, doi:10.1007/s11214-019-0614-x.

Warren, P. H. (1990), Am. Mineral., 75, 46-58.

Weider, S.Z., et al. (2014) Icarus, 235, 170-186.

Weider, S.Z., et al. (2015). Earth Planet. Sci. Lett., 416, 109-120.

Whitten, J. L., et al. (2014) Icarus, 241, p. 97-113.

Wood, J. A., et al. (1970), Proceedings of the Apollo 11 Lunar Science Conference, pp. 965-988. Wurm, G., et al. (2013), Astrophys. J., 769, article id. 78, 7 pp. 